Pacific Journal of Mathematics

SCATTERING FOR NON-LINEAR WAVE EQUATIONS

lix Earl Browner and Walter Strauss 


\section{SCATTERING FOR NON-LINEAR WAVE EQUATIONS}

\section{Felix E. Browder and Walter A. Strauss}

Introduction. Let $H$ be a Hilbert space, $A$ a positive densely defined self-adjoint linear operator in $H$ (i.e. $\left.A \geqq c_{0} I>0\right), M_{t}(u)$ a family of (possibly) non-linear operators with domain and range in $H$ and depending on the real parameter $t,-\infty<t<+\infty$.

Consider the generalized "wave equation"

$$
\frac{d^{2} u}{d t^{2}}(t)+(A u)(t)+M_{t}(u(t))=0
$$

where solutions are functions $u(t)$ from the real line $E^{1}$ to $H$. The equation (1) may obviously be regarded as a perturbation of the simpler equation

$$
\frac{d^{2} u}{d t^{2}}+A u=0
$$

The scattering problem for the perturbed equation (1) consists of the following:

(I). Let $u_{0}(t)$ be any solution of equation (2). For any real number $s$, prove the existence of a solution $u_{s}(t)$ of the perturbed equation (1) such that

$$
u_{s}(s)=u_{0}(s) ;\left(\frac{d u_{s}}{d t}\right)(s)=\left(\frac{d u_{0}}{d t}\right)(s) .
$$

(II). Show that as $s \rightarrow \pm \infty, u_{s}(t)$ converges in some suitable sense to solutions $u_{ \pm \infty}(t)$ of equation (2). In this case, we define $W_{-}\left(u_{0}\right)=u_{-\infty}(t) ; W_{+}\left(u_{0}\right)=u_{+\infty}(t)$.

(III). Study the properties of the operators $W_{-}$and $W_{+}$defined in (II), show the existence of $W_{+}^{-1} W_{-}=S$, and study the properties of the scattering operator $S$.

In a preceding paper [5], the second-named author has solved the scattering problem for equation (1) under the hypothesis that there exist a summable function $\theta(t)$ on $E^{1}$ such that

$$
\left\|A^{1 / 2}\left[M_{t}(u)-M_{t}(v)\right]\right\| \leqq \theta(t)\|A u-A v\|
$$

Receivẹ Mạch 28, 1962. 
for any pair of elements $u$ and $v$ in $D(A)$. This result was applied in [5] to the classical case of a perturbed wave equation in the Lorentz-invariant Klein-Gordon Hilbert space. A basic limitation of these results is the restricted non-linearity of $M_{t}(u)$, which for each $t$ must satisfy the uniform Lipschitz condition (4) in $u$.

In another preceding paper [2], the first-named author has given a study of the Cauchy initial value problem for the abstract wave equation (1) with $M_{t}(u)$ independent of $t$, and obtained therefrom a generalization of results of $K$. Jörgens [3] on the equation

$$
u^{\prime \prime}-\Delta u+m^{2} u+k u^{3}=0, \quad k \geqq 0, \quad m>0
$$

in $E^{3}$, as well as more general equations of the form

$$
u^{\prime \prime}-\Delta u+F^{\prime}\left(|u|^{2}\right) u=0
$$

in $E^{n}$. Here the non-linearity in $M(u)=M_{t}(u)$ was much less restricted but at the cost of definiteness assumptions to insure the positivity of the "energy" for solutions of (1) for all time."

It is our purpose in the present paper to use the methods of [2] to study the scattering problem for more strongly nonlinear perturbations $M_{t}(u)$ under appropriate positivity assumptions on $M_{t}(u)$. The prototype equation which we have in mind is the following modification of (5) on $E^{3}$,

$$
u^{\prime \prime}-\Delta u+m^{2} u+k(t) u^{3}=0
$$

with $k(t)$ summable and non-negative on $E^{1}$. We might equally as well think of

$$
u^{\prime \prime}-\Delta u+m^{2} u+k(t) F^{\prime}\left(|u|^{2}\right) u=0
$$

on $E^{n}$ with conditions depending upon $n$ imposed upon $F^{\prime}$. In general, the uniform Lipschitz condition (4) is replaced by the following:

Assumption I. For each $C>0$, there exists a summable function $k_{o}(t)$ such that for every pair of elements $u$ and $v$ of $D\left(A^{1 / 2}\right)$ with $\left\|A^{1 / 2} u\right\| \leqq C,\left\|A^{1 / 2} v\right\| \leqq C$, we have for all $t$ in $E^{1}$ :

$$
\begin{gathered}
\left\|M_{t}(u)\right\| \leqq k_{o}(t), \\
\left\|M_{t}(u)-M_{t}(v)\right\| \leqq k_{o}(t)\left\|A^{1 / 2}(u-v)\right\| .
\end{gathered}
$$

The other assumption which we shall make guarantees that to some degree the solutions of equation (1) behave as if they conserve a positive energy expression. Some such condition is necessary to

1 Added in proof. Results like those of [2] have also been obtained by I. E. Segal in a paper entitled "non-linear semigroups," to appear. 
obtain solutions of nonlinear wave equations with significant nonlinearity in the potential as shown by the examples given by J. S. Keller in [4]. The condition which we shall impose in the present paper is the following:

Assumption II. There exist constants $a, T_{0}>0$, a summable nonnegative numerical function $h(t)$ on $E^{1}$, and a nonnegative numerical function $G_{t}(u)$, defined for $u$ in $D\left(A^{1 / 2}\right)$ and bounded on bounded subsets of $D\left(A^{1 / 2}\right)$ in the graph norm, such that:

(1) For every continuously differentiable function $u(t)$ from $E^{1}$ to $D\left(A^{1 / 2}\right)$ and every $s$ in $E^{1}$,

$$
\left.\frac{d}{d t}\left\{G_{s}(u(t))\right\}\right|_{t=s}=2 \operatorname{Re}\left(M_{s}(u(s)), \frac{d u}{d t}(s)\right) .
$$

(2) The function $H_{t}(u)=d / d t\left\{G_{t}(u)\right\}$ exists for each fixed $u$ in $D\left(A^{1 / 2}\right)$ and satisfies the conditions

$$
\left|H_{t}(u)\right| \leqq h(t)\left\|A^{1 / 2} u\right\|^{2 a}
$$

and

$$
\begin{array}{ll}
H_{t}(u) \leqq 0, & t \leqq-T_{0} \\
H_{t}(u) \geqq 0, & t \geqq T_{0}
\end{array}
$$

(where we note explicitly that $T_{0}$ is independent of $u$ in $D\left(A^{1 / 2}\right)$ ).

(3) There exists a continuous function $c(t)$ on $E^{1}$ which is independent of $u$ and such that for all $t \in E^{1}, u \in D\left(A^{1 / 2}\right)$

$$
\left|H_{t}(u)\right| \leqq c(t)\left|G_{t}(u)\right| \text {. }
$$

Our basic result in the present paper is that the scattering problem in the sense described above, with a suitable definition of the solutions of our differential equations as well as the topology on these solutions, always has a solution under the assumptions (I) and (II). To obtain such a solution, we consider weak solutions of equations (1) and (2) rather than strict solutions. By choosing a different Hilbert space, however, each such weak solution can be considered as a strict solution in the new space. By making such a transformation, we identify our results with those of [5], where however, the corresponding results were obtained under different hypotheses and by a different argument.

Section 1 is devoted to the definition and study of weak solutions of equations (1) and (2), and contains the proof of the existence and uniqueness of weak solutions of the Cauchy initial value problem for equation (1). In $\S 2$, we apply these results to the solution of the 
scattering problem. In $\S 3$, we consider examples of equations (1) satisfying our assumptions.

Sections 1. Let $H$ be a Hilbert space, $A$ a positive densely defined self-adjoint linear operator in $H$. Set $B=A^{1 / 2}$, the positive square root of $A$. If $u(t)$ is a function from $E^{1}$ to $H$, we shall denote its first and second $t$-derivatives by $u^{\prime}(t)$ and $u^{\prime \prime}(t)$, respectively.

Consider the differential equation

$$
u^{\prime \prime}+A u=f(t)
$$

with the initial conditions

$$
u(0)=\Phi, \quad u^{\prime}(0)=\Psi .
$$

If $\Phi$ lies in $D(A), \Psi$ in $D(B)$, and if $B f(t)$ is defined for all $t$ and summable in $t$, there exists an unique strict solution for the equation (1.1) with the initial conditions (1.2), i.e. a twice-continuously differentiable solution $u(t)$ from $E^{1}$ to $H$ with $u(t) \in D(A)$ for each $t$ and $A u(t)$ continuous in $t$. This solution is given by

$$
u(t)=\cos (B t) \Phi+\sin (B t)\left(B^{-1} \Psi\right)+\int_{0}^{t} \sin (B(t-s))\left(B^{-1} f\right)(s) d s .
$$

If we make the weaker assumptions that $\Phi \in D(B), \Psi \in H$, and that $f(t)$ is locally summable in $t$, no such solution exists. However, the formula (1.3) continues to make sense and defines a continuously differentiable function $u(t)$ from $E^{1}$ to $H$ with $B u(t)$ defined for all $t$ and continuous in $t$. Thus we may define:

DEFINITION 1.1. The weak solution $u_{s, \Phi, y}(t)$ of equation (1.1) with initial conditions

$$
u(s)=\Phi, u^{\prime}(s)=\Psi,\left(s \in E^{1}, \phi \in D(B), \psi \in H,\right),
$$

is defined to be

$$
\begin{aligned}
u_{s, \Phi, y}(t)=\cos (B(t-s)) \Phi & +\sin (B(t-s))\left(B^{-1} \Psi\right) \\
& +\int_{s}^{t} \sin (B(t-r))\left(B^{-1} f\right)(r) d r .
\end{aligned}
$$

LEMMA 1.1 (a) Every weak solution $u=u_{s, \Phi, \Psi}$ is once continuously differentiable from $E^{1}$ to $H, u(t)$ lies in $D(B)$ for all $t$, and $B u(t)$ is continuous in $t$. Further $u(s)=\Phi, u^{\prime}(s)=\Psi$.

(b) If $u=u_{s, \Phi, \Psi}$ is the weak solution of equation (1.1) with initial conditions (1.4), then for any $T>s$, we have

$$
\sup _{s \leqq t \leqq T}\left\{\left\|u^{\prime}(t)\right\|+\|B u(t)\|\right\} \leqq 2\left\{\|B \Phi\|+\|\Psi\|+\int_{8}^{T}\|f(r)\| d r\right\}
$$


(c) If $s<s_{1}$, then $u_{s, \Phi, y}(t)$ is the weak solution of equation (1.1) with initial conditions $u\left(s_{1}\right)=u_{s, \Phi, \Psi}\left(s_{1}\right), u^{\prime}\left(s_{1}\right)=u_{s, \Phi, \Psi}^{\prime}\left(s_{1}\right)$.

(d) For any real numbers $t<t_{1}$ and $u=u_{s, \Phi, t}$, we have

$$
\begin{aligned}
\left\|u^{\prime}\left(t_{1}\right)\right\|^{2} & +\left\|B u\left(t_{1}\right)\right\|^{2}=\left\|u^{\prime}(t)\right\|^{2} \\
& +\|B u(t)\|^{2}+2 \operatorname{Re} \int_{t}^{t_{1}}\left(f(r), u^{\prime}(r)\right) d r .
\end{aligned}
$$

Proof of Lemma 1.1. Proof of (a). From formula (1.5), we have

$$
\begin{aligned}
u^{\prime}(t)=-\sin (B(t-s)) B \Phi & +\cos (B(t-s) \Psi \\
& +\int_{s}^{t} \cos (B(t-r)) f(r) d r,
\end{aligned}
$$

and

(1.9) $B u(t)=\cos (B(t-s)) B \Phi+\sin (B(t-s)) \Psi+\int_{s}^{t} \sin (B(t-r)) f(r) d r$.

Since $f$ is locally summable, $\Phi \in D(B)$, and $\Psi \in H$, it follows from (1.8) and (1.9) that $u^{\prime}$ and $B u$ are continuous functions of $t$. The fact that $u(s)=\Phi, u^{\prime}(s)=\Psi$ follows by substitution in (1.5) and (1.8).

Proof of (b). From equation (1.8), we have

$$
\left\|u^{\prime}(t)\right\| \leqq\|B \Phi\|+\|\Psi\|+\int_{s}^{t}\|f(r)\| d r,
$$

because $\|\cos (B t)\|,\|\sin (B t)\| \leqq 1$. Similarly, we obtain from equation (1.9),

$$
\|B u(t)\| \leqq\|B \Phi\|+\|\Psi\|+\int_{s}^{t}\|f(r)\| d r .
$$

The inequality of (b) follows obviously from the inequalities (1.10) and (1.11).

Proof of (c). It follows from the inequality (1.6) together with the obvious linearity of the weak solution $u_{s, \Phi, \Psi}$ in terms of $\Phi, \Psi$, and $f$, that if $u^{(1)}$ is the weak solution of the equation $u^{\prime \prime}+A u=f^{(1)}(t)$ with initial data $\left[\Phi^{(1)}, \Psi^{(1)}\right]$, then

$$
\begin{aligned}
& \sup _{s \leqq t \leqq T}\left\|u^{\prime}(t)-u^{(1) \prime}(t)\right\|+\left\|B u(t)-B u^{(1)}(t)\right\| \\
& \leqq 2\left\{\left\|B \Phi-B \Phi^{(1) \prime}\right\|+\left\|\Psi-\Psi^{(1)}\right\|\right. \\
&\left.+\int_{s}^{T}\left\|f(r)-f^{(1)}(r)\right\| d r\right\} .
\end{aligned}
$$

In qualitative terms, inequality (1.12) expresses the continuous depen- 
dence of the weak solutions upon the triples $[\Phi, \Psi, f(r)]$. Suppose now that we choose a sequence of triples $\left[\Phi_{k}, \Psi_{k}, f_{k}(r)\right]$ such that

$$
B \Phi_{k} \rightarrow B \Phi, \quad \Psi_{k} \rightarrow \Psi, \quad \int_{t}^{T}\left\|f_{k}(r)-f(r)\right\| d r \rightarrow 0,
$$

as $k \rightarrow \infty$. Then for the corresponding weak solutions $u_{k}(t) ; u_{k}^{\prime}(t)$ and $B u_{k}(t)$ will converge uniformly in $H$-norm as $k \rightarrow \infty$ to $u^{\prime}(t)$ and $B u(t)$, respectively. Suppose further that $\Phi_{k} \in D(A), \Psi_{k} \in D(B)$, and that $f_{k}(t) \in D(B)$ for $t \in[s, T]$ and that $B f_{k}(t)$ is summable on $[s, T]$. (We can always obtain such an approximating sequence since $D(B)$ is dense in $H, D(A)$ is dense in $D(B)$ in the graph-norm of $B$, and we can approximate $f(t)$ by a step function in $L^{1}([s, T], H)$ and then approximate the steps with arbitrary precision by elements of $D(B)$.) It follows from our provious remarks and from equation (1.5) that the corresponding weak solutions $u_{k}(t)$ are actually strict solutions of the given problem. Hence $u_{k}(t)$ is also the strict solution of the initial value problem for the equation $u^{\prime \prime}(t)+A u(t)=f_{k}(t)$ on the interval $\left[s_{1}, T\right]$ with initial conditions $u\left(s_{1}\right)=u_{k}\left(s_{1}\right), u^{\prime}\left(s_{1}\right)=u_{k}^{\prime}\left(s_{1}\right)$. However, $u_{k}\left(s_{1}\right) \rightarrow u\left(s_{1}\right), u_{k}^{\prime}\left(s_{1}\right) \rightarrow$ $u^{\prime}\left(s_{1}\right)$ by the inequality (1.12) as $k \rightarrow \infty$. Hence $u_{k}(t), u_{k}^{\prime}(t)$, and $B u_{k}(t)$ converge uniformly on $\left[s_{1}, T\right]$ to the corresponding terms for the weak solution $v$ of the equation $v^{\prime \prime}(t)+A v=f(t)$ with initial data $\left[u\left(s_{1}\right), u^{\prime}\left(s_{1}\right)\right]$ at $s_{1}$. But $u_{k}(t) \rightarrow u(t)$ on $[s, T]$, so that $u(t)=v(t)$ and (c) is proved.

Proof of $(d)$. Arguing as in the proof of (c), we know that any weak solution is the limit of strict solutions in the sense that we have a sequence $u_{k}(t)$ of twice continuously differentiable functions into $H$ satisfying the equations

$$
u_{k}^{\prime \prime}(t)+A u_{k}(t)=f_{k}(t)
$$

with $u_{k}(t)$ converging uniformly in norm to $u(t)$ on $\left[t, t_{1}\right], f_{k}$ converging to $f$ in $L^{1}\left(\left[t_{1}, t\right], H\right), u_{k}^{\prime}(t)$ coverging uniformly to $u^{\prime}(t)$, and $B u_{k}(t)$ converging uniformly to $B u(t)$. It suffices then to prove the equality (1.7) for each of the $u_{k}$ since the values of both sides of that equation for $u_{k}$ will converge as $k \rightarrow \infty$ to the corresponding values of $u$.

We may thus assume without loss of generality that our weak solution $u$ is actually a strict solution. Taking the inner product of both sides of the equation

$$
u^{\prime \prime}(t)+A u(t)=f(t)
$$

with $u^{\prime}(t)$, we obtain

$$
\frac{1}{2} \frac{d}{d r}\left\{\left(u^{\prime}(r), u^{\prime}(r)\right)+(B u(r), B u(r))\right\}=\operatorname{Re}\left(f(r), u^{\prime}(r)\right) .
$$


Integrating (1.13) from $t$ to $t_{1}$, we obtain

$$
\begin{aligned}
\left\|u^{\prime}\left(t_{1}\right)\right\|^{2} & -\left\|u^{\prime}(t)\right\|^{2}+\left\|B u\left(t_{1}\right)\right\|^{2}-\|B u(t)\|^{2} \\
& =2 \operatorname{Re} \int_{t}^{t_{1}}\left(f(r), u^{\prime}(r)\right) d r .
\end{aligned}
$$

This last equation is trivially equivalent to (1.7). Q.E.D.

Remark. Consider the Hilbert space $H^{\prime}=H \oplus D(B)$, with the norm $\|B u\|$ on $D(B)$. For $s, t \in E^{1}$, and a fixed locally summable function $f$ from $E^{1}$ to $H$, we may define $T(t, a)[\Phi, \Psi]=\left[u_{s}(t), u_{s}^{\prime}(t)\right]$, for $[\Phi, \Psi] \in H^{\prime}$. Then Lemma (1.1)(b) states that $T(t, s)$ is a bounded linear mapping of $H^{\prime}$ into $H^{\prime}$, while Lemma (1.1)(c) gives us the equation of evolution

$$
T(t, s)=T\left(t, s_{1}\right) T\left(s_{1}, s\right) .
$$

It follows that $T(t, s)=\{T(s, t)\}^{-1}$ since $T(s, s)$ is the identity map.

DEFINITION 1.2. Let $M_{t}(u)$ be a (possibly) nonlinear operator from $D(B)$ into $H$ for each $t$ in $E^{1}$. Suppose that $u$ is a function in $C^{1}([s, T], H)$ with $u(t) \in D(B)$ for all $t, B u \in C^{0}([s, T], H)$. Suppose further that $M(u(t)) \in L^{1}([s, T], H)$. Then $u$ is said to be a weak solution of

$$
u^{\prime \prime}(t)+A u(t)+M(u(t))=0, \quad s \leqq t \leqq T,
$$

with initial conditions

$$
u(s)=\Phi, u^{\prime}(s)=\Psi
$$

for given elements $\Phi \in D(B), \Psi \in H$, provided that

$$
\begin{aligned}
u(t)=\cos (B(t-s)) \Phi+\sin (B(r-s))\left(B^{-1} \Psi\right) \\
-\int_{s}^{t} M \sin (B(t-r))\left(B^{-1} M_{r}(u(r))\right) d r
\end{aligned}
$$

for $t \in[s, T]$.

In other terms, we have replaced our original differential equation with boundary conditions (1.15) by the single integral equation (1.16). We might ask about the relation of these weak solutions to the ordinary or strict solutions of equation (1.14), and an interesting remark in this direction is provided by the following simple result.

Proposition 1. Let $A$ and $M_{t}(u)$ be given as in Definition (1.2) and set $T_{t}(u)=B^{-1} M_{t}(u), \Phi_{1}=B^{-1} \Phi, \Psi_{1}=B^{-1} \Psi$. Then $u(t)$ is $a$ weak solution of equation (1.14) with initial conditions (1.15) if and only if $v(t)=B^{-1} u(t)$ is a strict solution of the equation 


$$
v^{\prime \prime}(t)+A v(t)+T_{t}(v(t))=0, \quad s \leqq t \leqq T
$$

with $v(s)=\Phi_{1}, v^{\prime}(s)=\Psi_{1}$.

Proof. If $u$ is given by equation (1.16), then applying $B^{-1}$ to both sides of that equation, we obtain

$$
\begin{aligned}
v(t)=\cos (B(t-s)) \Phi_{1}+\sin (B(t-s))\left(B^{-1} \Psi_{1}\right) \\
-\int_{s}^{t} \sin (B(t-r))\left(B^{-1} T_{r}(u(r)) d r .\right.
\end{aligned}
$$

It follows immediately by inspection of (1.18) that the latter implies that $v$ is a strict solution of the differential equation (1.17) with the initial data $\left[\Phi_{1}, \Psi_{1}\right]$ at $s$. Suppose, on the other hand, that $v(t)$ is a strict solution of equation (1.17) with the initial data $\left[\Phi_{1}, \Psi_{1}\right]$ at $s$. It follows from the argument of the beginning of $\S 1$ that $v$ must be a solution of the integral equation (1.17). Applying $B$ to both sides of equation (1.17), we find that $u(t)=B v(t)$ is a solution of equation (1.16), i.e. $u$ is a weak solution of the differential equation (1.14) with initial data $\left[B \Phi_{1}, B \Psi_{1}\right]$. Q.E.D.

REMARK. The correspondence given by Proposition 1 between weak solutions and strict solutions is the translation (in the linguistic sense) by which we can pass from the results of the present paper to results of the type given in [5]. In [5], it was assumed about the operator $T_{t}(u)$ that

$$
\left\|B\left(T_{t}(u)-T_{t}(v)\right)\right\| \leqq h(t)\|A u-A v\| ; u, v \in D(A), \text { all } t,
$$

with $h(t)$ a fixed summable function. Setting $M_{t}(u)=B T_{t}(u)$, we see that this is equivalent in our present notation to assuming that

$$
\left\|M_{t}(u)-M_{t}(v)\right\| \leqq h(t)\|B u-B v\|,
$$

a uniform Lipschitz condition on each $M_{t}$ as a mapping from $D(B)$ to $H$. The hypotheses of the present paper amount to replacing the uniform Lipschitz condition by the assumption that $M_{t}(u)$ is bounded and satisfies a Lipschitz condition on each bounded subset of $D(B)$ as a mapping into $H$.

We propose now to carry through the proof of the existence and uniqueness of weak solutions of the equation (1.14) with the initial conditions (1.15).

THEOREM 1. Let $H$ be a Hilbert space, $A$ a positive, densely defined linear operator in $H, B=A^{1 / 2}$. Let $M_{t}(u)$ be a (possibly) nonlinear operator from $D(B)$ to $H$ for $t$ in $E^{1}$ satisfying assump- 
tions (I) and (II). Then for every $\Phi$ in $D(B)$ and $\Psi$ in $H$ and for every locally summable function $f$ from $E^{1}$ to $H$, there exists one and only one weak solution of the equation

$$
u^{\prime \prime}(t)+A u+M_{t}(u)=0, \quad t \in E_{1}
$$

in the sense of Definition (1.2) where $u$ is a $C^{1}$ function from $E^{1}$ to $H$, and $u$ satisfies the initial conditions

$$
u(s)=\Phi, \quad u^{\prime}(s)=\Psi
$$

for a given $s$ in $E^{1}$.

Let us remark that for the existence and uniqueness of solutions, we can weaken our hypotheses in the following fashion: In Assumption (I), $k_{c}(t)$ may be merely summable. In Assumption (II), we may drop condition (2).

THEOREM 2. $u(t)=u_{s, \oplus, y}(t)$ be the weak solution described in Theorem 1 with initial data $[\Phi, \Psi]$ at $s$. Then we have

$$
\sup _{-\infty<t<+\infty}\left\{\left\|u^{\prime}(t)\right\|^{2}+\|B u(t)\|^{2}\right\}=k_{c}<+\infty
$$

where $k_{c}$ depends only on $C=\|\Psi\|^{2}+\|B \Phi\|^{2}$ and the data of Assumption $I$ and $I I$.

We begin the proof of Theorems 1 and 2 with the following Lemma which implies Theorem 2 and which will be an essential tool in the proof of Theorem 1.

LemMA (1.2). For each $C>0$, there exists a constant $k_{0}>0$ such that for any interval $\left[T_{1}, T_{2}\right]$ and for any weak solution $u$ of the equation

$$
u^{\prime \prime}+A u+M_{t}(u)=0
$$

on $\left[T_{1}, T_{2}\right]$ with

$$
\left\|u^{\prime}(s)\right\|^{2}+\|B u(s)\|^{2} \leqq C
$$

for a single point $s$ of $\left[T_{1}, T_{2}\right]$, we have

$$
\sup _{T_{1} \leqq t \leqq T_{2}}\left\{\|B u(t)\|^{2}+\left\|u^{\prime}(t)\right\|^{2}\right\} \leqq k_{o} .
$$

Proof of Lemma (1.2). By dividing up the interval $\left[T_{1}, T_{2}\right]$ into the two sub-intervals $\left[T_{1}, s\right]$ and $\left[s, T_{2}\right]$, it suffices to consider subintervals of the latter form. Our hypotheses on the equation (1.19) and especially on its nonlinear part $M_{t}(u)$ are invariant under a time- 
reversal, so that it suffices to consider only intervals of the form $\left[s, T_{2}\right]$, i.e. replace $T_{1}$ by $s$.

By Lemma (1.1) (d), since $u$ is a weak solution of equation (1.19), we know that for each $t$ in $\left[s, T_{2}\right]$,

$$
\left\|u^{\prime}(t)\right\|^{2}+\|B u(t)\|^{2} \leqq C-2 R e \int_{s}^{t}\left(M_{r}(u(r)), u^{\prime}(r)\right) d r .
$$

By assumption (II) on $M_{t}(u)$, we know that for every $r$

$$
2 \operatorname{Re}\left(M_{r}(u(r)), u^{\prime}(r)\right)=\frac{d}{d r}\left\{G_{r}(u(r))\right\}-H_{r}(u(r))
$$

where the functions $G$ and $H$ satisfy the conditions of Assumption (II). Therefore, we obtain from equation (1.22) the following:

$$
\begin{aligned}
\left\|u^{\prime}(t)\right\|^{2} & +\|B u(t)\|^{2}+G_{r}(u(t)) \leqq C+G_{s}(u(s)) \\
& +\int_{s}^{t} H_{r}(u(r)) d r .
\end{aligned}
$$

Since $G_{r}(u)$ is uniformly bounded on bounded subsets of $D(B)$, it follows that $C+G_{s}(u(s)) \leqq C^{\prime}$, the latter being a constant which depends only on $C$ and the function $G$.

Let

$$
E(t)=\left\|u^{\prime}(t)\right\|^{2}+\|B u(t)\|^{2}+G_{t}(u(t)) .
$$

It follows from (1.24) that

$$
E(t) \leqq C^{\prime}+\int_{s}^{t} H_{r}(u(r)) d r .
$$

From Assumption (II), we know three inequalities on the nonlinear functional $H_{r}(u)$. We know that for $r \geqq T_{0}, H_{r}(u(r)) \leqq 0$. We know also that

$$
\left|H_{r}(u(r))\right| \leqq c(r)\left|G_{r}(u(r))\right| \leqq c(r) E(r),
$$

with a continuous function $c(r)$ independent of $u$, while

$$
\left|H_{r}(u(r))\right| \leqq h(r)\|B u(r)\|^{2 a} \leqq h(r)[E(r)]^{a} .
$$

From the first of these inequalities and inequality (1.25), it follows that for $t \geqq T_{0}$,

$$
E(t) \leqq C^{\prime}+\int_{s}^{T_{0}} H_{r}(u(r)) d r .
$$

Hence it suffices to bound $E(t)$ on the interval [ $s, T_{0}$ ], i.e. to assume that $T_{2}=T_{0}$. Choose an arbitrary point $p$ within the interval $\left[s, T_{0}\right]$, with the choice of $p$ to be restricted later, and estimate the right- 
hand side of (1.25) by using (1.27) on the interval $[s, p]$ and (1.27) on the complementary sub-interval $\left[p, T_{0}\right]$.

Consider the interval $[s, p]$ first. Using inequality (1.27) we have

$$
E(t) \leqq C^{\prime}+\int_{s}^{t} h(r)[E(r)]^{a} d r, \quad s \leqq t \leqq p .
$$

Set

$$
v(t)=\int_{s}^{t} h(r)[E(r)]^{a} d r .
$$$$
s \leqq t \leqq p
$$

It follows from (1.28) that

$$
v^{\prime}(t) \leqq h(t)\left[C^{\prime}+v(t)\right]^{a}, \quad v(s)=0 .
$$

while

$$
E(t) \leqq C^{\prime}+v(t) .
$$

To bound $E(t)$ on $[s, p]$, it thus suffices to bound $v(t)$. To do this, we shall apply the following elementary analytical result:

LEMMA 1.3. Let $v(t)$ be a nonnegative continuously differentiable function on the interval $[s, p]$ with $v(s)=0$ which satisfies the inequality

$$
v^{\prime}(t) \leqq h(t)\left[c_{0}+v(t)\right]^{a}, \quad s \leqq t \leqq p .
$$

with $c_{0}$ and $h$ a summable function on $E^{1}$ given constants. Then:

(i) If $a \leqq 1$,

$$
v(t) \leqq k\left(c_{0}, a\right), \quad s \leqq t \leqq p .
$$

with $k\left(c_{0}, a\right)$ independent of $v, s$, or $p$.

(ii) If $a>1$, then for each $c_{0}>0$, there exists $p\left(c_{0}\right)$ independent of $s$ such that

$$
v(t) \leqq k\left(c_{0}, a\right), \quad s \leqq t \leqq p \leqq p\left(c_{0}\right)
$$

Proof of Lemma (1.3). Proof of (i). We consider the case $a<1$ first. Then setting $w(t)=v(t)+c_{0}$, we have

$$
[w(t)]^{-a} w^{\prime}(t) \leqq h(t),
$$

or integrating from $s$ to $t$,

$$
[w(t)]^{1-a}-c_{0}^{1-a} \leqq(1-a) \int_{s}^{t} h(r) d r \leqq(1-a) \int_{-\infty}^{+\infty} h(r) d r,
$$

from which the sought conclusion follows for $w(t)$ and hence for $v(t)$. 
If $a=1$, we have

$$
\frac{d}{d t}[\log w(t)] \leqq h(t),
$$

and integrating,

$$
w(t) \leqq c_{0} \exp \left(\int_{-\infty}^{+\infty} h(r) d r\right) .
$$

which again justifies the conclusion.

Proof of (ii). If $a>1$, it follows from the inequality

$$
w^{\prime}(t)[w(t)]^{-a} \leqq h(t)
$$

that

$$
(1-a)^{-1}\left[w(t)^{1-a}-c_{0}^{1-a}\right] \leqq \int_{8}^{t} h(r) d r \leqq \int_{-\infty}^{p} h(r) d r .
$$

By an elementary calculation, we have

$$
[w(t)]^{a-1} \leqq\left[c_{0}^{1-a}-(a-1) \int_{-\infty}^{p} h(r) d r\right]^{-1} .
$$

If we choose $p\left(c_{0}\right)$ such that

$$
\int_{-\infty}^{p^{\left(c_{0}\right)}} h(r) d r \leqq(2 a-2)^{-1} c_{0}^{1-a}
$$

we then have

$$
[w(t)]^{a-1} \leqq 2 c_{0}^{a-1}, \quad s \leqq t \leqq p \leqq p\left(c_{0}\right),
$$

i.e.

$$
w(t) \leqq c_{0} a^{(a-1)^{-1}},
$$

and the conclusion of Lemma (1.3) is established.

Proof of Lemma (1.2) continued. We apply Lemma (1.3) to the function $v(t)$ defined in terms of $E(t)$ above. By Lemma (1.3) (ii), there exists $p_{0}$, depending only upon $c_{0}=C^{\prime}$ and hence ultimately only upon $C$ and not upon $s$, or $u$, such that for $p \leqq p_{0}, v(t)$ is uniformly bounded by $k(c, a)$ on the interval $[s, p]$ by virtue of (1.28) or (1.29). (If $s$ is greater than $p_{0}$, we choose $p=s$. If $s$ is less than $p_{0}$, we choose $p=p_{0}$.) On the interval $\left[p, T_{0}\right]$, we know that the function $H_{r}(u(r))$ satisfies the inequality (1.26) where the function $c(r)$ being continuous is bounded from above by a constant $C_{0}$. This constant $C_{0}$ may be taken as $\max _{p_{0} \leqq t \leqq T_{0}} c(r)$, and hence depends only 
upon $C$. Therefore, we have the inequality for $p_{0}<t \leqq T_{0}$,

$$
E(t) \leqq C^{\prime \prime}+C_{0} \int_{p}^{t} E(r) d r, \quad\left(C^{\prime \prime}=E(p)\right) .
$$

Setting

$$
v(t)=C^{\prime \prime}+C_{0} \int_{p}^{t} E(r) d r
$$

we have

$$
v^{\prime}(t) \leqq C_{0} v(t),\left(p \leqq t \leqq T_{0}\right) ; \quad v(p)=C^{\prime \prime}
$$

Hence

$$
v(t) \leqq C^{\prime \prime} \exp \left(C_{0}(t-p)\right) \leqq C^{\prime \prime} \exp \left(C_{0}\left(T_{0}-p_{0}\right)\right), \quad p \leqq t \leqq T_{0} .
$$

Thus we have obtained uniform bounds (depending on $C$ but not on $s$ or $u$ ) for the function $v(t)$ on the interval $\left[s, T_{0}\right]$ from which a uniform bound for $E(t)$ on $\left[s, T_{2}\right]$ for any $T_{2} \geqq T_{0}$ follows by our previous remarks. Since $G_{r}(u(r))$ is assumed nonnegative by Assumption (II), $E(t)$ dominates $\left\|u^{\prime}(t)\right\|^{2}+\|B u(t)\|^{2}$ and the proof of Lemma (1.2) is complete.

Lemma 1.4. For each $C>0$, there exists $k_{0}>0$ such that if $u(t)$ and $u_{1}(t)$ are weak solutions of $u^{\prime \prime}(t)+M_{t}(u(t))=0$ on any interval $\left[T_{1}, T_{2}\right], s$ any point of $\left[T_{1}, T_{2}\right]$, then

$$
\begin{gathered}
\sup _{T_{1} \leqq t \leqq T_{2}}\left\{\left\|u^{\prime}(t)-u_{1}^{\prime}(t)\right\|^{2}+\left\|B u(t)-B u_{1}(t)\right\|^{2}\right\} \\
\leqq k_{o}\left\{\left\|u^{\prime}(s)-u_{1}^{\prime}(s)\right\|^{2}+\left\|B u_{1}(s)\right\|^{2}\right\}
\end{gathered}
$$

provided that

$$
\left\|u^{\prime}(s)\right\|^{2}+\|B u(s)\|^{2} \leqq C, \quad\left\|u_{1}^{\prime}(s)\right\|^{2}+\left\|B u_{1}(s)\right\|^{2} \leqq C .
$$

Proof of Lemma (1.4). Since time reversal preserves our hypotheses, we may reduce the general case as before to the case of the interval $\left[s, T_{2}\right]$, i.e. take $T_{1}=s$. By Lemma (1.2), there exists a constant $k_{o}^{\prime}$ such that

$$
\begin{aligned}
& \sup _{s \leqq t \leqq T_{2}}\left\{\left\|u^{\prime}(t)\right\|^{2}+\|B u(t)\|^{2}\right\} \leqq k_{o}^{\prime}, \\
& \sup _{s \leqq t \leqq T_{2}}\left\{\left\|u_{1}^{\prime}(t)\right\|^{2}+\|B u(t)\|^{2}\right\} \leqq k_{o}^{\prime} .
\end{aligned}
$$

Let $w=u-u_{1}$. Then $w(t)$ is a weak solution of the equation

$$
w^{\prime \prime}(t)+A w(t)=f(t)
$$

with 


$$
f(t)=M_{t}\left(u_{1}(t)\right)-M_{t}(u(t)) .
$$

By Assumption (I) on $M_{t}(u)$ together with the bounds established on $B u(t)$ and $B u_{1}(t)$, there exists a summable function $h_{o}(t)$ such that

$$
\|f(t)\| \leqq h_{o}(t)\left\|B u_{1}(t)-B u(t)\right\|=h_{o}(t)\|B w(t)\| .
$$

Applying the inequality (1.6) of Lemma (1.1) (b) to $w(t)$ on the interval $[\mathrm{s}, t], t \leqq T_{2}$, we find from (1.34) that if we set

$$
F(t)=\left\|w^{\prime}(t)\right\|+\|B w(t)\|,
$$

then

$$
F(t) \leqq C_{1}+2 \int_{s}^{t} h_{o}(r) F(r) d r .
$$

Setting $v(t)=C_{1}+2 \int_{s}^{t} h_{o}(r) F(t) d r$, we find that $v(t)$ satisfies the differential inequality

$$
v^{\prime}(t) \leqq 2 h_{c}(t) v(t)
$$

with $v(s)=C_{1}$. Hence for all $t \geqq s$ for which (1.37) holds, and in particular on $\left[s, T_{2}\right]$, we have

$$
v(t) \leqq C_{1} \exp \left(2 \int_{s}^{t} h(r) d r\right) \leqq C_{1} \exp \left(2 \int_{-\infty}^{+\infty} h(r) d r\right)=C_{2} .
$$

The constant $C_{1}$ depends only on $C$, and therefore so does $C_{2}$. From (1.36), we know that $F(t) \leqq v(t) \leqq C_{2}$, and since it follows trivially that $F(t)$ bounds a fixed multiple of $\left\|w^{\prime}(t)\right\|^{2}+\|B w(t)\|^{2}$, the proof of Lemma (1.4) is complete.

Corollary to Lemma (1.4). Any weak solution $u(t)$ of $u^{\prime \prime}+$ $A u+M_{t}(u)=0$ on an interval $\left[T_{1}, T_{2}\right]$ is uniquely determined by the pair $\left[u(s), u^{\prime}(s)\right]$ for any single point $s$ in $\left[T_{1}, T_{2}\right]$.

LEMmA 1.5. It suffices in order to prove Theorem 1 to show that for each $C>0$, there exists $d_{o}>0$ such that for any real $T$ and any pair $[\Phi, \Psi]$ with $\Phi \in D(B), \Psi \in H$ with $\|B \Phi\|^{2}+\|\Psi\|^{2} \leqq C$, there exists a weak solution of the equation

$$
u^{\prime \prime}(t)+A u(t)+M_{t}(u(t))=0
$$

on the interval $\left[T, T+d_{o}\right]$ with the initial data

$$
u(T)=\Phi, \quad u^{\prime}(T)=\Psi .
$$

Proof of Lemma (1.5). Let $\Phi$ and $\Psi$ be given with $\Phi \in D(B)$, 
$\Psi \in H$, and set $C=\|B\|^{2}+\|\Psi\|^{2}$. Let $s$ be a given real number. By symmetry under time-reversal, it suffices to show that there exists a solution of equation (1.37) with $u(s)=\Phi, u^{\prime}(s)=\Psi$ on the infinite half-line $[s, \infty]$. By the Corollary to Lemma (1.4), it suffices to show that for every $T>s$, there exists a solution of (1.37) on $[s, T]$ with the given initial values at $s$. Indeed for $T_{1}>T$, the two solutions obtained on the intervals $[s, T]$ and $\left[s, T_{1}\right]$, respectively, must coincide on $[s, T]$. We may therefore amalgamate all the solutions on the various intervals starting at $s$ to form a single weak solution over the interval $[s, \infty]$.

Suppose that Theorem 1 were false. Then there would have to exist a real number $T>s$ such that no weak solution existed on the interval $[s, T]$. We shall deduce a contradiction. By the hypothesis of Lemma (1.5), there would exist a solution on some interval $\left[s, s+d_{\sigma}\right]$ with $d_{\sigma}>0$. Consider all weak solutions $u(t)$ of equation (1.37) on intervals of the form $\left[s, T_{1}\right], T_{1}>s$, with $u(s)=\Phi, u^{\prime}(s)=\Psi$, for the given $\Phi$ and $\Psi$. By the uniqueness Corollary to Lemma (1.4), all such solutions would coincide on the overlap of their intervals of definition. Taking the union of the intervals of definition, we obtain a weak solution $u(t)$ of equation (1.37) on a half-open interval [s, $T_{0}$ ], $T_{0} \leqq T$, with the property that $u$ is not continuable as a weak solution beyond $T_{0}$. By Lemma (1.2), however, there exists a constant $k_{\sigma}$ independent of $u$ or $T_{0}$ such that

$$
\sup _{s \leqq t \leqq T_{0}}\left\{\left\|u^{\prime}(t)\right\|^{2}+\|B u(t)\|^{2}\right\} \leqq k_{C} .
$$

Consider $k_{o}=C_{1}$ as the constant $C$ of the hypothesis of Lemma (1.5). Then there exists $d>0$ such that for every $T_{2}<T_{0}$ a weak solution $u_{1}(t)$ of equation (1.37) exists with initial conditions

$$
u_{1}\left(T_{2}\right)=u\left(T_{2}\right), \quad u^{\prime}\left(T_{2}\right)=u^{\prime}\left(T_{2}\right)
$$

on the interval $\left[T_{2}, T_{2}+d\right]$. Taking $T_{2}$ in the interval $\left(T_{0}-d, T_{0}\right)$, we have a weak solution $u_{1}(t)$ of $(1.37)$ on an interval $\left[T_{2}, T_{0}+\varepsilon\right]$ with $\varepsilon>0$ having the same Cauchy data at $T_{2}$ as $u(t)$ itself. By the uniqueness theorem, $u_{1}(t)=u(t), u(t)$ is continuable beyond $T_{0}$, and we have a contradiction establishing the conclusion of Lemma (1.5).

Proof of Theorem 1. It suffices by Lemma (1.5) to prove the existance on a small interval $\left[s, s+d_{\sigma}\right]$ of a weak solution $u(t)$ of

$$
u^{\prime \prime}(t)+A u(t)+M_{t}(u(t))=0
$$

with initial values $u(s)=\Phi, u^{\prime}(s)=\Psi$, under the assumption

$$
\|B \Phi\|^{2}+\|\Psi\|^{2} \leqq C \text {. }
$$


To carry through the local existence proof, we fix $s, \Phi$ and $\Psi$ and, choosing $d>0$, we construct a complete metric space $X$ depending on $s, \Phi, \Psi$, and $d$ as follows: $X$ consists of all functions $u$ from the interval $[s, s+d]$ into $H$ having the following properties:

(a) $u$ is once continuously differentiable from $[s, s+d]$ into $H$.

(b) $B u(t)$ is defined for all $t$ in $[s, s+d]$ and the function $B u$ is continuous from $[s, s+d]$ into $H$,

(c) $u(s)=\Phi, u^{\prime}(s)=\Psi$.

The distance function on $X$ is given by

$$
\left(u, u_{1}\right)=\left\|u-u_{1}\right\|_{x}=\sup _{s \leqq t \leqq s+d}\left\{\left\|B u(t)-B u_{1}(t)\right\|+\left\|u^{\prime}(t)-u_{1}^{\prime}(t)\right\|\right\} .
$$

The completeness of $X$ in this metric follows by standard arguments.

Once $X$ is given as above, we construct a mapping $S$ of $X$ into itself whose fixed points (if any) will be the desired weak solutions $u$ of equation (1.37) with the given initial condition. If $v \in X, w=S(v)$ is defined by

$$
\begin{gathered}
w(t)=\cos (B(t-s)) \Phi+\sin (B(t-s))\left(B^{-1} \Psi\right)-\int_{s}^{t} \sin (B(t-r)) \\
B^{-1}\left\{M_{r}(v(r))\right\} d r, \quad(s \leqq t \leqq s+d) .
\end{gathered}
$$

Thus $w=S(v)$ is the weak solution on $[s, s+d]$ of the equation

$$
w^{\prime \prime}(t)+A w(t)=-M_{t}(v(t))
$$

with initial values $w(s)=\Phi, w^{\prime}(s)=\Psi$.

We shall show that for a suitable $C_{1}>C$, and for $d$ sufficiently small, the mapping $S$ maps the nonempty closed set $\|v\|_{X} \leqq C_{1}$ into itself and that on that set, $S$ is a contraction mapping with constant less than one, i.e.

$$
\left\|S v-S v_{1}\right\|_{x} \leqq c\left\|v-v_{1}\right\|_{x} c<1 ;\|v\|_{x}, \quad\left\|v_{1}\right\|_{x} \leqq C_{1} .
$$

By the Picard contraction principle, $S$ will have a fixed point in that set, but a fixed point $v$ of $S$ is precisely a weak solution of equation (1.37) on the interval $[s, s+d]$ with the prescribed initial conditions. Since, moreover, the restriction on the size of $d$ will depend only upon the constant $C$, the conclusion of Theorem 1 will then follow from Lemma (1.5).

To establish the facts cited in the preceding argument, we apply the conclusions of Lemma (1.1). We know that $w=S(v)$ is a weak solution of $w^{\prime \prime}+A w=f(t)$, where $f(t)=-M_{t}(v(t))$. Since

$$
\|f(t)\|=\left\|M_{t}(v(t))\right\| \leqq h(t)\|v\|_{x},
$$


we obtain from Lemma (1.1) (b),

$$
\|w\|_{x} \leqq 2\|B \Phi\|+2\|\Psi\|+2\|v\|_{x} \int_{s}^{s+a} h(r) d r \leqq C_{1}+o(d)\|v\|_{X},
$$

where $C_{1}$ and the function of $d$ written as $o(d)$ may be chosen independent of $s$ and $v$, and $C_{2}$ depends upon $C$. If $\|v\|_{x} \leqq C_{1}$, then $\|w\|_{X} \leqq C_{2}+o_{\alpha}(1) C_{1}$, and if $C_{1}$ is chosen initially so that $C_{1} \geqq 2 C_{2}$, we may choose $d$ so small that for $d \leqq d_{0},\|S(v)\|_{x} \leqq C_{1}$. Thus we have for small enough $d>0$, a nonempty closed set $\|v\|_{X} \leqq C_{1}$ mapped by $S$ into itself.

Now let $v$ and $v_{1}$ be two elements of the set $\|v\|_{X} \leqq C_{1}$ in $X$, and let $w=S(v), w_{1}=S\left(v_{1}\right)$. We wish to estimate $\left\|w-w_{1}\right\|_{x}$ in terms of $\left\|v-v_{1}\right\|_{x}$. Let $y(t)=w(t)-w_{1}(t)$. Then $y$ is a weak solution of the equation

$$
y^{\prime \prime}(t)+A y(t)=M_{t}\left(v_{1}(t)\right)-M_{t}(v(t))=g(t),
$$

with $y(s)=0, y^{\prime}(s)=0$.

By Lemma (1.1) (b), we know therefore that

$$
\|y\|_{x} \leqq 2 \int_{s}^{s+a}\|g(r)\| d r
$$

while

$$
\begin{aligned}
\|g(r)\| & =\left\|M_{r}\left(v_{1}(r)\right)-M_{r}(v(r))\right\| \leqq k_{\sigma_{1}}(r)\left\|B v_{1}(r)-B v(r)\right\| \\
& \leqq k_{\sigma_{1}}(r)\left\|v-v_{1}\right\|_{X},
\end{aligned}
$$

where the second inequality follows from Assumption (I) on the nonlinear operator $M_{t}(u) . \quad C_{1}$ is now fixed, and $k_{\sigma_{1}}(r)$ is therefore a prescribed summable function of $r$ independent of $s$ or $v$ but depending only upon $C_{1}$. We have then

$$
\|y\|_{x} \leqq 2\left\|v-v_{1}\right\|_{x} \int_{s}^{s+a} k_{\sigma_{1}}(r) d r \leqq o_{\alpha}(1)\left\|v-v_{1}\right\|_{X} .
$$

Choosing $d \leqq d_{0}$ (dependent only upon $C_{1}$ and hence upon $C$ ), we may ensure that $o_{\alpha}(1) \leqq 1 / 2$, and thereby the proof of Theorem 1 is complete.

As we have remarked ealier, Theorem 2 is an immediate consequence of Lemma (1.2) so that our discussion of the existence and uniqueness of weak solution of the nonlinear wave equation (1) is therefore complete.

Sections 2. The scattering problem. As it was stated in the Introduction, the scattering problem for the nonlinear wave equation (1) has three parts, (I), (II), and (III), We have completed part (I) 
in the preceding section. Let us now carry through a discussion of parts (II) and (III), at least to the extent of showing the existence of the wave operators $W_{-}$and $W_{+}$under our given hypotheses and of the scattering operator $S$. We leave the more detailed discussion of the properties of $S$ to another place.

Let $H_{0}$ be the set of weak solutions on $E^{1}$ of the equation

$$
u^{\prime \prime}(t)+A u(t)=0,
$$

and $H_{M}$ the set of weak solutions on $E^{1}$ of the nonlinear equation

$$
u^{\prime \prime}(t)+A u(t)+M_{t}(u(t))=0 .
$$

By Lemma (1.2) and Theorem 2, we know that for every $u$ in $H_{M}$, we have

$$
\sup _{t}\left\{\|B u(t)\|^{2}+\left\|u^{\prime}(t)\right\|^{2}\right\}<+\infty,
$$

and the same fact is obviously true for elements $u_{0}$ of $H_{0}$.

DEFINITION 2.1. $H_{0}$ and $H_{M}$ are made into metric spaces where the metric is given by

$$
p\left(u, u_{1}\right)=\sup _{t}\left[\left\|B\left(u-u_{1}\right)(t)\right\|^{2}+\left\|u^{\prime}(t)-u_{1}^{\prime}(t)\right\|^{2}\right]^{1 / 2} .
$$

DEFINITION 2.2. For any real $s, W_{s}$ is the mapping of $H_{0}$ into $H_{M}$ defined by $W_{s}\left(u_{0}\right)=u$, if and only if $u$ is the solution of equation (1) with $u(s)=u_{0}(s), u^{\prime}(s)=u_{0}^{\prime}(s)$.

As observed in [5], for a suitable choice of the Hilbert space $H$ in the case where $A$ is $(-\Delta)+m^{2}, H_{0}$ is the so called Klein-Gorden Hilbert space of quantum field theory.

LEMma 2.1. (a) $H_{0}$ and $H_{M}$ are complete metric spaces. Each $W_{s}$ maps $H_{0}$ onto $H_{M}$.

(b) Given $C>0$ and $\varepsilon>0$, there exists $t_{0}$ independent of $s$ such that if $\left\|u_{0}\right\|_{B_{0}} \leqq C$, then $\left\|W_{s}\left(u_{0}\right)-W_{s_{1}}\left(u_{0}\right)\right\|_{H_{M}}<\varepsilon$ for $s, s_{1} \leqq-t_{0}$ (or for $\left.s, s_{1} \geqq t_{0}\right)$.

(c) Given $C>0$, there exists $k_{0}>0$ such that for $u_{0}, v_{0}$ in $H_{0}$ with $\left\|u_{0}\right\|_{H_{0}} \leqq C$, then

$$
\left\|W_{s}\left(u_{0}\right)-W_{s}\left(v_{0}\right)\right\|_{B_{M}} \leqq k_{\sigma}\left\|u_{0}-v_{0}\right\|_{B_{0}} .
$$

(d) Let $W_{s}^{-1}=T_{s}$, be the inverse mapping of $H_{M}$ into $H_{0}$. Then the assertions of (b) and (c) hold with $W_{s}$ replaced by $T_{s}$ and $H_{0}$ replaced by $H_{M}$.

(c) If $\left\|u_{0}\right\|_{B_{0}} \leqq c$, then $\left\|W_{s}\left(u_{0}\right)\right\|_{H_{M}} \leqq k_{\sigma}$ for all s. If $\|u\|_{H_{M}} \leqq C$, 
$\left\|T_{s}(u)\right\|_{H_{0}} \leqq k_{\sigma}$.

Proof of Lemma (2.1). Proof of (a). The compleness of $H_{0}$ and $H_{M}$ follows immediately from the definition of weak solution together with Theorems 1 and 2 . Since a weak solution $u$ of either equation (1) or equation (2) exists for any $\Phi$ in $D(B)$ and $\Psi$ in $H$ with $u(s)=\Phi$ and $u^{\prime}(s)=\Psi$ and is uniquely determined by the pair $[\Phi, \Psi]$, it follows that $W_{s}$ maps all weak solutions of equation (2) onto all weak solutions of equation (1), i.e. each $W_{s}$ maps $H_{0}$ onto $H_{M}$.

Proof of $(b)$. Since $\left\|u_{0}\right\|_{H} \leqq C$, it follows from Theorem 2 that for all $s,\left\|W_{s}\left(u_{0}\right)\right\|_{H_{M}} \leqq k_{o}$. Let $s$ and $s_{1}$ be given, and let $u=W_{s}\left(u_{0}\right)$, $u_{1}=W_{s_{1}}\left(u_{0}\right)$. Let $w=u_{1}-u_{0}$. Suppose $s<s_{1}$. On the interval $[s, s]$, $w$ is a weak solution of the equation

$$
w^{\prime \prime}(t)+A w(t)=-M_{t}\left(u_{1}(t)\right) .
$$

At $s_{1}, w$ satisfies the initial conditions $w\left(s_{1}\right)=0, w^{\prime}\left(s_{1}\right)=0$. Hence by Lemma (1.1) (b), on the whole interval $\left[s, s_{1}\right]$ and in particular at $s, w$ satisfies the inequality

$$
\|B w(t)\|+\left\|w^{\prime}(t)\right\| \leqq 2 \int_{t}^{s_{1}}\left\|M_{r}\left(u_{1}(r)\right)\right\| d r .
$$

By Assumption (I) on $M_{t}(u)$ and by the bound on the norm of $u_{1}$, we know that

$$
\left\|M_{r}\left(u_{1}(r)\right)\right\| \leqq h_{o}(r)\left\|u_{1}\right\|_{H_{0}} \leqq k_{o} h_{o}(r)
$$

where $h_{\sigma}$ is a summable function dependent upon $C$ but independent of $s$ and $s_{1}$. Hence

$$
\|B w(s)\|+\left\|w^{\prime}(s)\right\| \leqq k_{o}^{\prime} \int_{s}^{s_{1}} h_{o}(r) d r .
$$

By choosing both $s$ and $s_{1}$ sufficiently negative (or sufficiently positive) we can therefore insure that

$$
\|B w(s)\|^{2}+\left\|w^{\prime}(s)\right\|^{2}<\delta
$$

for any given $\delta>0$. But $w(s)=u_{1}(s)-u_{0}(s)$,

$$
w^{\prime}(s)=u_{1}^{\prime}(s)-u_{0}^{\prime}(s), \quad B w(s)=B u_{1}(s)-B u_{0}(s) .
$$

Applying Lemma (1.4) and choosing $\delta$ sufficiently small, we can therefore insure that $\left\|u_{1}-u\right\|_{H_{M}}=\left\|W_{s_{1}}\left(u_{0}\right)-W_{s}\left(u_{0}\right)\right\|_{H_{M}}<\varepsilon$.

Proof of (c). The conclusion of (c) follows from Lemma (1.4) together with the fact that $\left\|W_{s}\left(u_{0}\right)\right\|_{H_{M}}$ is uniformly bounded for 
all $s$.

Proof of $(d)$. The conclusions of (d) follows by the same arguments as those of (b) and (c) if one replaces equation (1) by equation (2).

Proof of $(e)$. We have already observed these facts in the course of the preceding argument.

THEOREM 3. As $s \rightarrow+\infty, W_{s}$ as a sequence of operators from $H_{0}$ to $H_{H}$ converges uniformly on bounded subsets of $H_{0}$ to an operators $W_{ \pm}$mapping $H_{0}$ one-to-one and onto $H_{M}$ which satisfies a Lipschitz condition on bounded sets of $H_{0}$ and maps bounded sets into bounded sets. Similarly $W_{s}$ converges to an operator $W_{-}$as $s \rightarrow-\infty$, where $W_{-}$maps $H_{0}$ one-to-one and onto $H_{\mu}$, satisfies a Lipschitz condition on each bounded set of $H_{0}$, and maps bounded sets of $H_{\mu}$. Both $W_{+}$and $W_{-}$have inverse $T_{+}$and $T_{-}$defined on $H_{M}$ which satisfy Lipschitz conditions on bounded sets and map bounded sets into bounded sets.

Corollary to Theorem 3. If $S=W_{+}^{-1} W_{-}, S$ is a one-to-one continuous mapping of $H_{0}$ onto itself such that $S$ and its inverse map bounded sets into bounded sets and satisfy a Lipschitz condition on each bounded subset of $H_{0}$.

Proof of Theorem 3. It follows from Lemma (2.1) (b) that $W_{s}$ converges uniformly on bounded subsets of $H_{0}$ as $s \rightarrow+\infty$, or $-\infty$. By Lemma (2.1) (c), the limits $W_{+}$and $W_{-}$satisfy Lipschitz conditions on each bounded set of $H_{0}$. By Lemma (2.1) (e), they map bounded subsets of $H_{0}$ into bounded subsets of $H_{M}$. By Lemma (2.1) (d), $T_{s}=W_{s}^{-1}$ converges similarly to $T_{+}$and $T_{-}$as $s \rightarrow+\infty$ or $-\infty$, respectively. For each $s$,

$$
\begin{array}{ll}
\left(W_{s} T_{s}\right)(u)=u, & u \in H_{M} \\
\left(T_{s} W_{s}\right)\left(u_{0}\right)=u_{0}, & u \in \mathrm{H}_{0} .
\end{array}
$$

It follows immediately that $W_{-} T_{-}$and $W_{+} T_{+}$are the identity on $H_{M}$, and that $T_{-} W_{-}$and $T_{+} W_{+}$are the identity on $H_{0}$. The conclusion of the theorem is then complete.

Since $T_{s} W_{ \pm}$converges strongly to the identity operator on $H_{0}$ as $s \rightarrow \pm \infty$, the wave operators exist in the sense of [5].

Section 3. Examples and remarks. To obtain examples of operators $A$ and $M_{t}(u)$ for which the Assumptions (I) and (II) hold, we 
let $H=L^{2}\left(E^{n}\right)$ where $E^{n}$ is the $n$-dimensional Euclidean space, and let $A$ be the unique self-adjoint realization of a uniformly eliptic differential self-adjoint operator of order $2 m$ with coefficients uniformly continuous on $E^{n}$. (cf. [1]). We assume that $A$ is positive. Let $M_{t}(u)=g(t) F^{\prime}\left(|u|^{2}\right) u$, where $F(r)$ is a twice-continuously differentiable function on $E^{1}$. Then Assumptions (I) and (II) will be verified under the following conditions:

(a) $F(0)=0, F(r) \geqq 0, g(t)$ is continuously differentiable. For $r>1, F^{\prime}(r) \leqq r^{q}$, where $q=m(n-2 m)^{-1}$ (or any finite number if $n \leqq 2 m), F^{\prime \prime}(r) \leqq r^{q-1}$ for $r \geqq 1$.

(b) $g(t)>0$ for all $t ; g^{\prime}(t) \geqq 0$ for $t \leqq-T_{0}$ and $g^{\prime}(t) \leqq 0$ for $t \geqq T_{0}$. Further, $g$ lies in $L^{1}\left(E^{1}\right)$.

The essential parts of the verification are given in $\S 2$ of [2]. Slight variants of the above condition (b) may be given and in particular, one in which $h(t)$ is nonnegative and vanishes outside a bounded interval. (In this last case the second part of the scattering problem is trivial).

Theorem 1, 2 and 3 may be generalized under suitable hypotheses to the case where $A$ is not self-adjoint or depends upon the time parameter $t$.

\section{BIBLIOGRAPHY}

1. F. E. Browder, On the spectral theory of elliptic differential operators, I, Math. Annalen, 142 (1961), 22-130.

2. - On non-linear wave equations, Math. Zeitschr., 80 (1962), 249-264.

3. K. Jorgens, Das Anfangswertproblem im Grossen fur eine Klasse nichtlinearer Wellengleichungen, Math. Zeitschr., 77 (1961), 295-308.

4. J. B. Keller, On solutions of nonlinear wave equations, Comm. Pure. App. Math, 10 (1957), 523-530.

5. W. A. Strauss, Scattering for hyperbolic equations, M. I. T. Doctoral Dissertation, Jan. 1962. 



\section{PACIFIC JOURNAL OF MATHEMATICS}

\section{EDITORS}

\section{RalPh S. Phillips}

Stanford University

Stanford, California

M. G. Arsove

University of Washington

Seattle 5, Washington
J. Dugundji

University of Southern California Los Angeles 7, California

Lowell J. Paige

University of California

Los Angeles 24, California

\section{ASSOCIATE EDITORS}
E. F. BECKENBACH
D. DERRY
H. L. ROYDEN
E. G. STRAUS
T. M. CHERRY
M. OHTSUKA
E. SPANIER
F. WOLF

\section{SUPPORTING INSTITUTIONS}

\author{
UNIVERSITY OF BRITISH COLUMBIA \\ CALIFORNIA INSTITUTE OF TECHNOLOGY \\ UNIVERSITY OF CALIFORNIA \\ MONTANA STATE UNIVERSITY \\ UNIVERSITY OF NEVADA \\ NEW MEXICO STATE UNIVERSITY \\ OREGON STATE UNIVERSITY \\ UNIVERSITY OF OREGON \\ OSAKA UNIVERSITY \\ UNIVERSITY OF SOUTHERN CALIFORNIA
}

STANFORD UNIVERSITY

UNIVERSITY OF TOKYO

UNIVERSITY OF UTAH

WASHINGTON STATE UNIVERSITY

UNIVERSITY OF WASHINGTON

AMERICAN MATHEMATICAL SOCIETY

CALIFORNIA RESEARCH CORPORATION SPACE TECHNOLOGY LABORATORIES

NAVAL ORDNANCE TEST STATION

Printed in Japan by International Academic Printing Co., Ltd., Tokyo Japan 


\section{Pacific Journal of Mathematics}

\section{Vol. 13, No. 1 \\ March, 1963}

Frantz Woodrow Ashley, Jr., A cone of super-(L) functions............. 1

Earl Robert Berkson, Some metrics on the subspaces of a Banach space....

Felix Earl Browder and Walter Strauss, Scattering for non-linear wave

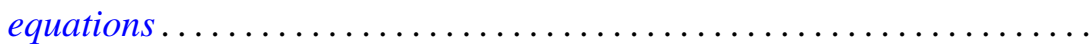

Edmond Darrell Cashwell and C. J. Everett, Formal power series ..........

Frank Sydney Cater, Continuous linear functionals on certain topological

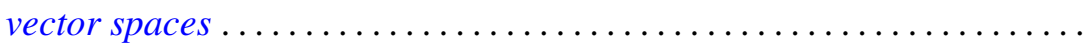

John Douglas Dixon, General group extensions ....................

Robert Pertsch Gilbert, On harmonic functions of four variables with

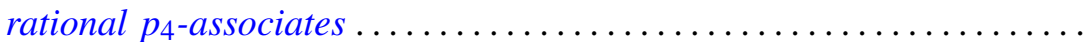

Irving Leonard Glicksberg, On convex hulls of translates ..............

Simon Hellerstein, On a class of meromorphic functions with deficient zeros

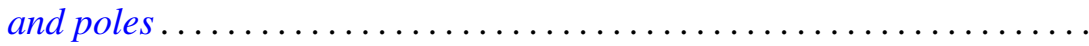

Donald William Kahn, Secondary cohomology operations which extend the

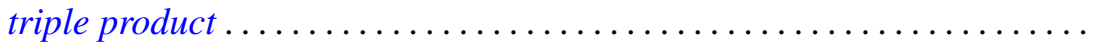

G. K. Leaf, A spectral theory for a class of linear operators .............

R. Sherman Lehman, Algebraic properties of the composition of solutions of partial differential equations ........................... 157

Joseph Lehner, On the generation of discontinuous groups ............. 169

S. P. Lloyd, On certain projections in spaces of continuous functions ...... 171 Fumi-Yuki Maeda, Generalized spectral operators on locally convex spaces ..................................

Donald Vern Meyer, $E^{3}$ modulo a 3-cell

William H. Mills, An application of linear programming to permutation groups.

Richard Scott Pierce, Centers of purity in abelian groups

Christian Pommerenke, On meromorphic starlike functions ...

Zalman Rubinstein, Analytic methods in the study of zeros of

polynomials...

B. N. Sahney, On the Nörlund summability of Fourier series

Tôru Saitô, Regular elements in an ordered semigroup . .

Lee Meyers Sonneborn, Level sets on spheres...........

Charles Andrew Swanson, Asymptotic estimates for limit point

problems .

Lucien Waelbroeck, On the analytic spectrum of Arens . .

Alvin (Murray) White, Singularities of a harmonic function of three

variables given by its series development .............

Kōichi Yamamoto, Decomposition fields of difference sets ...

Chung-Tao Yang, On the action of $\mathrm{SO}(3)$ on a cohomology manifold... 